\title{
Impact of symptoms of anxiety and depression on COPD Assessment Test scores
}

Anxiety and depression are common comorbidities in patients with chronic obstructive pulmonary disease (COPD) and affect patients' health status $[1,2]$. Health status in COPD patients is frequently assessed by the St George's Respiratory Questionnaire (SGRQ), which is a validated disease-specific questionnaire widely used in clinical trials [3], but complex and time-consuming to complete and score. Consequently, the COPD Assessment Test (CAT) has been developed [4]. This is a disease-specific health status questionnaire containing eight items with good sensitivity, reliability and responsiveness [4]. CAT scores are strongly positively correlated with SGRQ scores in patients with COPD [4]. Therefore, it is reasonable to hypothesise that those COPD patients with symptoms of anxiety and/or depression will report higher CAT scores (equalling worse health status) compared to those without symptoms.

The latest version of the Global Initiative for Chronic Obstructive Lung Disease (GOLD) document [5] recommends the CAT as a tool to assess the impact of COPD on the individual patient beyond airflow limitation. Although it was known that clinically relevant symptoms of anxiety and depression are more common in patients in GOLD stage IV compared to patients in GOLD stages I and II [1], the frequency distribution of patients with symptoms of anxiety and depression across the updated GOLD classification remains unknown. Recently, SiLLEN et al. [6] reported a prevalence of symptoms of anxiety and depression of $38 \%$ and $32 \%$, respectively, in COPD patients with GOLD group D.

We aimed to assess the impact of symptoms of anxiety and depression on disease-specific health status as assessed by CAT in patients with COPD entering pulmonary rehabilitation. Secondarily, we aimed to investigate the relationship between the updated GOLD classification and symptoms of anxiety and depression.

Demographic and clinical characteristics (number of exacerbations in the last 12 months, current medication, post-bronchodilator spirometry and functional exercise capacity as assessed by 6-min walk distance) were assessed in stable patients with COPD entering pulmonary rehabilitation at the Centre of Expertise for Chronic Organ Failure (CIRO+), Horn, the Netherlands, and LHL Health Røros, Røros, Norway. Patients were eligible if they had a primary diagnosis of COPD [5] and were clinically stable. Patients were excluded if they had a history of other lung diseases, had undergone lung surgery or had malignancy within the last 5 years. The Regional Committee for Medical and Health Research Ethics in Central Norway (REK Midt 2012/1495) and the Medical Ethical Commission of the Maastricht University Medical Centre+ (MUMC+), Maastricht, the Netherlands (METC 11-3-070) approved this study.

Symptoms of anxiety and depression were assessed using the Hospital Anxiety and Depression Scale (HADS) [7]. The scores for each subscale range from 0 (optimal) to 21 (worst) points. A score $\geqslant 10$ points on the anxiety (HADS-A) or depression (HADS-D) subscale may indicate the presence of clinically relevant symptoms [7]. Disease-specific health status was assessed using the CAT [4]. The score ranges from 0 (no impact) to 40 points (severe impact) [4].

In total, 307 patients were included (51\% male, age $63.9 \pm 8.6$ years, with forced expiratory volume in $1 \mathrm{~s}$ of $50 \pm 21 \%$ predicted). In general, the sample was slightly overweight $\left(26.5 \pm 6.1 \mathrm{~kg} \cdot \mathrm{m}^{-2}\right)$ and functional exercise capacity $(434 \pm 132 \mathrm{~m})$ as well as disease-specific health status (CAT $21.1 \pm 6.5$ points) were impaired. $38(12.4 \%)$ patients reported clinically relevant symptoms of anxiety, 28 (9.1\%) patients reported clinically relevant symptoms of depression, and $50(16.3 \%)$ patients reported a combination of both symptoms. There were no differences in HADS-D scores between patients treated with antidepressants $(n=57)$ compared to patients not treated with antidepressants $(n=250 ; 7.0 \pm 4.4$ points versus $6.1 \pm 4.3$ points, $\mathrm{p}=0.13)$. Patients treated with anxiolytics $(\mathrm{n}=47)$ reported higher HADS-A scores compared to patients not treated with anxiolytics $(n=260 ; 10.0 \pm 5.1$ points versus $6.6 \pm 4.1$ points, $\mathrm{p} \leqslant 0.001)$.

Patients without symptoms of anxiety and/or depression had lower CAT scores (19.6 \pm 6.0 points) than patients with symptoms of anxiety $(22.8 \pm 6.2$ points, $\mathrm{p}=0.020)$, symptoms of depression $(24.8 \pm 6.3$ points, $\mathrm{p}<0.001)$ or both symptoms $(23.2 \pm 8.8$ points, $\mathrm{p}=0.002)$ (fig. 1$)$. CAT scores were modestly correlated with HADS-A scores $(\mathrm{r}=0.30, \mathrm{p}<0.001)$ and HADS-D scores $(\mathrm{r}=0.35, \mathrm{p}<0.001)$. There were no differences 
a)

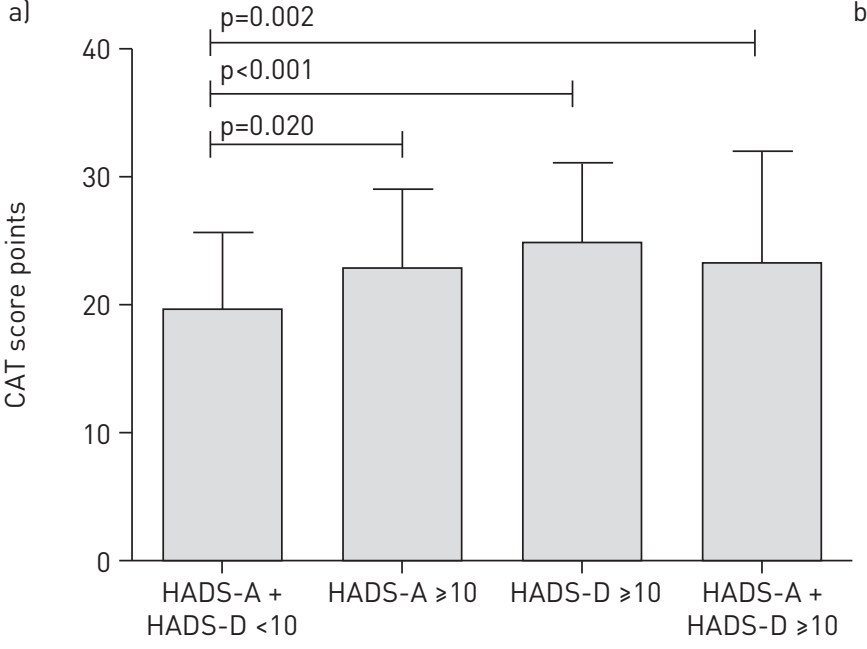

b)

\begin{tabular}{lccc}
\hline GOLD group & Patients $n$ & HADS-D $\geqslant 10$ points & HADS-A $\geqslant 10$ points \\
\hline A & 6 & 16.7 & 16.7 \\
B & 76 & 27.6 & 17.1 \\
C & 119 & 32.8 & 27.7 \\
D & 98 & 25.5 & 28.6 \\
\hline
\end{tabular}

FIGURE 1 a) Chronic obstructive pulmonary disease Assessment Test (CAT) scores, stratified by Hospital Anxiety and Depression Scale (HADS) scores. b) Proportions of patients reporting anxiety and depression, stratified by updated Global Initiative for Chronic Obstructive Lung Disease (GOLD) groups, given as percentages reporting clinically relevant symptoms. HADS-A: anxiety subscale; HADS-D: depression subscale. A HADS score $\geqslant 10$ points may indicate the presence of clinically relevant symptoms. $\mathrm{p}>0.05$ for comparisons between all GOLD groups.

in the proportions of patients with clinically relevant symptoms of anxiety and/or depression between GOLD groups A to D (fig. 1).

The current study has two novel findings: 1) COPD patients with symptoms of anxiety and/or depression referred for pulmonary rehabilitation reported a worse disease-specific health status as assessed by CAT than patients without these symptoms; 2) the prevalence of symptoms of anxiety and depression was equally distributed across the updated GOLD groups.

Lou et al. [2] reported that SGRQ scores were comparable between patients with symptoms of anxiety and patients with symptoms of depression. Our study shows comparable findings for symptoms of anxiety or depression and CAT scores. Patients hospitalised for a COPD exacerbation with depressive symptoms (using Beck's Depression Inventory) reported higher CAT scores (worse health status) than patients without depressive symptoms [8]. The current study extends these findings by adding that symptoms of anxiety and/ or depression affect patients' health status in stable patients.

In the present study, $37.8 \%$ of the patients had clinically relevant symptoms of anxiety and/or depression. This frequency is comparable to previous findings [1,2]. Antidepressants were more widely used than anxiolytics in our data. This might be explained by the fact that antidepressants have mixed effects, and that symptoms of anxiety and depression often coincide [9]. HADS-D scores were similar between patients treated with antidepressants and untreated patients, while patients treated with anxiolytics had higher HADS-A scores compared to those untreated. Psychological screening for anxiety and depression could have enlightened these findings, as a HADS score of $\geqslant 10$ points indicates the presence of symptoms of anxiety and depression but does not necessarily indicate a disorder [7].

JANSSEN et al. [1] identified a higher proportion of patients with clinically relevant symptoms of anxiety and depression in GOLD stage IV compared to patients in GOLD I and II. Recently, LEE et al. [10] reported a significantly higher proportion of patients with depression in GOLD groups B and D compared to GOLD groups A and C and concluded that "the frequency of depression differed by current symptoms, but not by the severity of the airway limitation." However, these results are not directly comparable with our findings since depression was assessed by the patient health questionnaire (PHQ)-9 [10]. In the present study, symptoms of anxiety and depression were equally distributed across the updated GOLD stages, indicating that the new classification does not capture clinically relevant symptoms of anxiety and depression. Accordingly, psychological symptoms should be assessed in individual COPD patients entering pulmonary rehabilitation, irrespective of GOLD group.

To conclude, patients with COPD with symptoms of anxiety and/or depression report worse diseasespecific health status as assessed by CAT than patients without these symptoms. Symptoms of anxiety and depression are equally distributed across the updated GOLD groups in COPD patients referred for pulmonary rehabilitation. 
Christina W. Hilmarsen ${ }^{1,9}$, Sarah Wilke ${ }^{2,9}$, Harald Engan ${ }^{1,3}$, Martijn A. Spruit ${ }^{2}$, Johan Rodenburg ${ }^{1}$, Daisy J.A. Janssen ${ }^{2}$, Sigurd Steinshamn ${ }^{1,4,5}$, Paul W. Jones ${ }^{6}$, Emiel F.M. Wouters ${ }^{2,7}$, Line Oldervoll ${ }^{1,8,10}$ and Frits M.E. Franssen ${ }^{2,10}$

${ }^{1}$ LHL Health Røros, Norwegian Heart and Lung Patient Organization, Røros, ${ }^{4}$ Lung Dept, St Olav's University Hospital, Trondheim, ${ }^{5}$ K.G. Jebsen Center of Exercise in Medicine, Dept of Circulation and Medical Imaging, Norwegian University of Technology and Science, Trondheim, and ${ }^{8}$ Centre for Health Promotion, Institute for Social Work and Health Science, Norwegian University of Technology and Science, Trondheim, Norway. ${ }^{2}$ Program Development Centre, CIRO+, Centre of Expertise for Chronic Organ Failure, Horn, and ${ }^{7}$ Dept of Respiratory Medicine, Maastricht University Medical Centre+ (MUMC+), Maastricht, The Netherlands. ${ }^{3}$ Dept of Engineering and Sustainable Development, Mid Sweden University, Östersund, Sweden. ${ }^{6}$ Division of Clinical Science, St George's University of London, London, UK. ${ }^{9}$ Both authors contributed equally. ${ }^{10}$ Both authors contributed equally.

Correspondence: C.W. Hilmarsen, Research and Development Group, LHL Helse Røros, Øverhagaen 15, N-7374 Røros, Norway. E-mail: cwhi@lhl-helse.no

Received: Sept 182013 | Accepted: Sept 182013 | First published online: Oct 102013

Support statement: The collection of the Dutch data was financially supported by the Lung Foundation Netherlands (3.4.10.015) and GlaxoSmithKline (SCO115406).

Conflict of interest: Disclosures can be found alongside the online version of this article at www.erj.ersjournals.com

\section{References}

Janssen DJ, Spruit MA, Leue C, et al. Symptoms of anxiety and depression in COPD patients entering pulmonary rehabilitation. Chron Respir Dis 2010; 7: 147-157.

2 Lou P, Zhu Y, Chen P, et al. Prevalence and correlations with depression, anxiety, and other features in outpatients with chronic obstructive pulmonary disease in China: a cross-sectional case control study. BMC Pulm Med 2012; 12: 53 .

3 Jones PW, Quirk FH, Baveystock CM, et al. A self-complete measure of health status for chronic airflow limitation. The St. George's Respiratory Questionnaire. Am Rev Respir Dis 1992; 145: 1321-1327.

4 Jones PW, Harding G, Berry P, et al. Development and first validation of the COPD Assessment Test. Eur Respir J 2009; 34: 648-654.

5 Vestbo J, Hurd SS, Agusti AG, et al. Global strategy for the diagnosis, management and prevention of chronic obstructive pulmonary disease: GOLD executive summary. Am J Respir Crit Care Med 2013; 187: 347-365.

6 Sillen MJ, Franssen FM, Delbressine JM, et al. Heterogeneity in clinical characteristics and co-morbidities in dyspneic individuals with COPD GOLD D: findings of the DICES trial. Respir Med 2013; 107: 1186-1194.

Zigmond AS, Snaith RP. The hospital anxiety and depression scale. Acta Psychiatr Scand 1983; 67: 361-370.

Papaioannou AI, Bartziokas K, Tsikrika S, et al. The impact of depressive symptoms on recovery and outcome of hospitalised COPD exacerbations. Eur Respir J 2013; 41: 815-823.

9 Kunik ME, Roundy K, Veazey C, et al. Surprisingly high prevalence of anxiety and depression in chronic breathing disorders. Chest 2005; 127: 1205-1211.

10 Lee YS, Park S, Oh YM, et al. Chronic obstructive pulmonary disease assessment test can predict depression: a prospective multi-center study. J Korean Med Sci 2013; 28: 1048-1054.

\section{Molecular epidemiological analysis suggests cross-infection with Pseudomonas aeruginosa is rare in non-cystic fibrosis bronchiectasis}

\section{To the Editor:}

In both cystic fibrosis (CF) and non-CF bronchiectasis (NCFBr) chronic Pseudomonas aeruginosa infection is adversely prognostic $[1,2]$. In $\mathrm{CF}$, epidemic infections with specific clones of $P$. aeruginosa are associated with further adverse outcomes [3,4]. This cross-infection risk has led to segregation of patients [5]. There are few data on $P$. aeruginosa cross-infection in NCFBr. As a result, segregation in NCFBr has not been addressed in guidelines [6]. 